\title{
Antepartum myomectomy: a possible procedure for successful outcome
}

\author{
Sabita Sai Chandran ${ }^{1,2 *}$, Sunita Samal ${ }^{1}$
}

\begin{abstract}
${ }^{1}$ Department of Obstetrics \& Gynaecology, Mahatma Gandhi Medical College \& Research Institute, Puducherry, India
${ }^{2}$ Department of Obstetrics \& Gynaecology, Indira Gandhi Medical College \& Research Institute, Puducherry, India
\end{abstract}

Received: 6 July 2014

Accepted: 13 July 2014

\section{*Correspondence:}

Dr. Sabita Sai Chandran,

E-mail: sabita.kutty@gmail.com

(C) 2014 Chandran SS et al. This is an open-access article distributed under the terms of the Creative Commons Attribution Non-Commercial License, which permits unrestricted non-commercial use, distribution, and reproduction in any medium, provided the original work is properly cited.

\begin{abstract}
Though myomas usually remain asymptomatic during pregnancy, in one third of the cases they may increase in size and develop red degeneration. Majority of the cases will respond to medical management and rarely require surgical intervention. Site, size and number of the fibroids and the placental position are the important factors to be considered before intervention. Our case was having fibroid with placental attachment at its base and had features of rapid growth with red degeneration not responding to medical management and hence myomectomy was done in second trimester. In properly evaluated and carefully selected patients the procedure will be successful and improve the pregnancy outcome.
\end{abstract}

Keywords: Degeneration, Leiomyoma, Management, Myomectomy, Pregnancy

\section{INTRODUCTION}

Myomas are the most common pelvic tumors complicating pregnancy. The incidence of myomas during pregnancy varies from $2-15 \%$ depending on maternal age. ${ }^{1}$ Many of these fibroids remain asymptomatic and require no treatment. However, 10$40 \%$ of these cases will have complications related to the effects of myoma on pregnancy. ${ }^{1-3}$ Majority of the fibroids do not change their size during pregnancy except in one third of cases in which they may grow especially during the first trimester and also to some extent in the second trimester. Red degeneration is the most common effect of pregnancy on fibroid and it occurs usually in large myomas presenting as acute abdominal pain. In rare cases myoma requires surgical intervention such as myomectomy during pregnancy and delivery. There are many reports on successful myomectomy being done during cesarean in the literature. However myomectomy performed during pregnancy remains as a rare entity. We report a case of non-pedunculated subserous myoma complicating pregnancy with rapid growth presented as recurrent abdominal pain not responding to medical management. Myomectomy was done during pregnancy with successful outcome.

\section{CASE REPORT}

A thirty four year old primi, married for two years attended our outpatient department at 11 weeks four days of gestation with a report of ultrasonography stating as eight weeks of pregnancy with adnexal mass. She presented with the history of abdominal pain for two weeks. Incidentally she also reported having undergone operation for cataract at the age of five years. Family history revealed the information that her mother had hypertension.

On general physical examination there was no abnormality except pallor. Examination of the abdomen revealed uterine size of 22 weeks of pregnancy with a large mass arising from the uterus in the fundal region. The mass was irregular in surface having varying consistency and tender on deep palpation. Ultrasound examination showed fibroid in the posterior fundal 
region. She was initially treated with anti-inflammatory agents. But she consulted often for recurrent episodes of abdominal pain and tenderness over the mass. There was no history of fever or vomiting. After the period of gestation of 14 weeks, ultrasound examination showed a non-pedunculated subserous fibroid with broad base at the site of placental implantation in the posterior fundal region and clinical examination revealed the uterine size reaching 28 weeks. Since the mass was found growing rapidly and she was also suffering from worsening abdominal pain, the decision for surgical management was taken. Abdomen was opened through midline incision extending above the umbilicus. The fibroid with a diameter of $15 \mathrm{~cm}$ with broad base arising from the body of the uterus in the posterior surface was found as shown in the figure. Myomectomy was done successfully with no intra-operative as well as post-operative events. The histopathology report confirmed fibroid with red degeneration. She was followed up in the antenatal outpatient department and was admitted after the completion of 34 weeks for safe confinement. Elective cesarean was performed at term and a live female baby with good APGAR, weighing $2.5 \mathrm{~kg}$ was delivered. The post-operative period was uneventful.

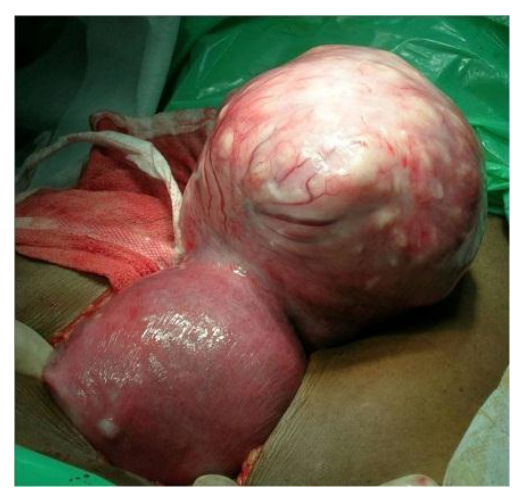

Figure 1: Intra-operative picture of pregnant uterus with fibroid.

\section{DISCUSSION}

Myomectomy during pregnancy is generally contraindicated or limited to tumors with a discrete pedicle that can be clamped and easily ligated. Resection of intramural myomas during pregnancy, or at the time of delivery, may stimulate profuse bleeding requiring hysterectomy. The size, location, and number of fibroids and their relation to the placenta are critical factors to be considered. Indications for myomectomy during pregnancy includes severe abdominal pain due to torsion of subserous pedunculated myomas or red degeneration not responding to medical treatment, and an increase in myoma size causing abdominal discomfort. Ultrasound scanning has a pivotal role in diagnosing and monitoring fibroids during pregnancy and in determining the relative position of the fibroids to the placenta. Contrary to clinical tenet, two studies ${ }^{4,5}$ showed that myomas do not grow during pregnancy. In our case the indication for surgery was persistent abdominal pain with a provisional diagnosis of red degeneration not responding to medical treatment and also the rapidly increasing size of the fibroid. Burton et al. in their case series of myomectomy in pregnancy considered only pedunculated myoma with base of the stalk not wider than five centimeter as the indication for removal. ${ }^{6}$ Though the scan measured the size of the base of the fibroid to be as large as five centimeter in diameter located in the implantation region, we could perform the operation with no complications. She was operated in the second trimester which will rule out the possibility of malformations as mentioned in the literature. ${ }^{7}$ Our experience makes us to suggest that myomectomy during pregnancy may be considered safe in selected cases. But the current data available represent a small sample population to conclude the related safety and risk. Further investigations are needed to evaluate the safety of performing myomectomy during pregnancy.

\section{Funding: No funding sources \\ Conflict of interest: None declared \\ Ethical approval: Not required}

\section{REFERENCES}

1. Katz VL, Dotters DJ, Droegemueller W. Complications of uterine leiomyomas in pregnancy. Obstet Gynaecol. 1989;73:593-6.

2. Rice JP, Kay HH, Mahony BS. The clinical significance of uterine leiomyomas in pregnancy. Am J Obstet Gynaecol. 1989;160:1212-6.

3. Michalas SP, Oreopoulou FV, Papageorgiou JS. Myomectomy during pregnancy and caesarean section. Hum Reprod. 1995;10:1869-70.

4. Muram D, Gillieson M, Walters JH. Myomas of the uterus in pregnancy: ultrasonographic follow-up. Am J Obstet Gynaecol. 1980;138:16-9.

5. Winer-Muram HT, Muram D, Gillieson MS, Ivey BJ, Muggah HF. Uterine myomas in pregnancy. Can Med Assoc J. 1981;128: 949-50.

6. Burton CA, Grimes DA, March CM. Surgical management of leiomyomata during pregnancy. Obstet Gynaecol. 1989;74:707-9.

7. Joo JG, Inovay J, Silhavy M and Papp Z. Successful enucleation of a necrotizing fibroid causing oligohydramnios and fetal postural deformity in the 25th week of gestation: a case report. J Reprod Med. 2001;46:923-5.

DOI: $10.5455 / 2320-1770 . i j \operatorname{rog} 20140947$

Cite this article as: Chandran SS, Samal S.

Antepartum myomectomy: a possible procedure for successful outcome. Int J Reprod Contracept Obstet Gynecol 2014;3:818-9. 